\title{
Cloud detection for MIPAS using singular vector decomposition
}

\author{
J. Hurley, A. Dudhia, and R. G. Grainger \\ Atmospheric, Oceanic and Planetary Physics, Clarendon Laboratory, Department of Physics, Parks Road, Oxford, UK
}

Received: 31 March 2009 - Published in Atmos. Meas. Tech. Discuss.: 29 April 2009

Revised: 7 September 2009 - Accepted: 8 September 2009 - Published: 18 September 2009

\begin{abstract}
Satellite-borne high-spectral-resolution limb sounders, such as the Michelson Interferometer for Passive Atmospheric Sounding (MIPAS) onboard ENVISAT, provide information on clouds, especially optically thin clouds, which have been difficult to observe in the past. The aim of this work is to develop, implement and test a reliable cloud detection method for infrared spectra measured by MIPAS.

Current MIPAS cloud detection methods used operationally have been developed to detect cloud effective filling more than $30 \%$ of the measurement field-of-view (FOV), under geometric and optical considerations - and hence are limited to detecting fairly thick cloud, or large physical extents of thin cloud. In order to resolve thin clouds, a new detection method using Singular Vector Decomposition (SVD) is formulated and tested. This new SVD detection method has been applied to a year's worth of MIPAS data, and qualitatively appears to be more sensitive to thin cloud than the current operational method.
\end{abstract}

\section{Introduction}

Clouds are increasingly recognised for their influence on the radiative balance of the Earth and the implications that they have on possible climate change, as well as in air pollution and acid-rain production. However, clouds remain a major source of uncertainty in climate models. High thin clouds such as cirrus are important to study.

High clouds are frequently observed at all latitudes and, at any one time, $60 \%$ of the Earth's surface is covered by cirrus (Wylie et al., 2005). High clouds are important because they are high enough to act to warm the Earth; however this mechanism is not well understood in terms of the

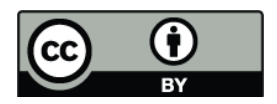

Correspondence to: J. Hurley

(hurley@atm.ox.ac.uk) relation of micro- and macro-physical cloud properties. Because they are so wide-spread and permanent, it is important to understand how these clouds affect the climate. However, current cloud detection algorithms often miss much thin cloud in satellite measurements, and the instrumentation itself (eg. nadir versus limb, microwave versus infrared and so on) specifies varying thresholds of senstivity to different cloud types - and hence conventional cloud climatologies and inventories are incomplete with respect to high thin cloud such as cirrus (Wylie et al., 2005).

There have been many studies on clouds over the years and many climatologies: by Barton (1983), Warren et al. (1985), Woodbury and McCormick (1983), Prabhakara et al. (1988), Wylie and Menzel (1989), Wylie et al. (1994) - but these were all limited by a lack of global coverage. Currently, the Stratospheric Aerosol and Gas Experiment (SAGE) (e.g. SAGE, 2002), High Resolution Infrared Radiation Sounder (HIRS) instrument (e.g. Wylie et al., 2005), International Satellite Cloud Climatology Project (ISCCP) (e.g. ISCCP, 2008) and GRAPE project (e.g. Sayer et al., 2009) are actively compiling cloud climatologies.

\section{Overview of MIPAS-ENVISAT}

The Michelson Interferometer for Passive Atmospheric Sounding (MIPAS) is an infrared limb-viewing instrument and was launched in March 2002 on the European Space Agency's Environmental Satellite (ENVISAT). ENVISAT is in an $800 \mathrm{~km}$ sun-synchronous polar orbit, with a nominal orbit having a repeat period of 35 days, an orbital period of $100.6 \mathrm{~min}$ and an inclination of $98.54^{\circ}$. The inclination of the orbit in conjunction with azimuth scanning enables full global coverage pole-to-pole (ESA, 2005).

MIPAS was designed to measure limb-emission spectra (primarily for retrievals of temperature, pressure and trace gases such as $\mathrm{CO}_{2}, \mathrm{O}_{3}, \mathrm{H}_{2} \mathrm{O}, \mathrm{HNO}_{3}, \mathrm{CH}_{4}, \mathrm{~N}_{2} \mathrm{O}$

Published by Copernicus Publications on behalf of the European Geosciences Union. 
and $\left.\mathrm{NO}_{2}\right)$ at a high spectral resolution in the near- to mid-infrared from $685 \mathrm{~cm}^{-1}$ to $2410 \mathrm{~cm}^{-1}$ in five discrete bands (A $685-970 \mathrm{~cm}^{-1}$, AB $1020-1170 \mathrm{~cm}^{-1}$, B $1215-$ $1500 \mathrm{~cm}^{-1}$, C $1570-1750 \mathrm{~cm}^{-1}$, and D $1820-2410 \mathrm{~cm}^{-1}$ ). In its initial operating specifications, MIPAS operated at a spectral resolution of $0.025 \mathrm{~cm}^{-1}$, measuring spectra nominally every $3 \mathrm{~km}$ vertically in the troposphere - however following persistent slide malfunctions in early 2004, the resolution was decreased to $0.0625 \mathrm{~cm}^{-1}$ but the measurement tangent height spacing decreased to nominally every $1.5 \mathrm{~km}$ in the lower stratosphere and troposphere (Mantovani, 2005).

The FOV of MIPAS is approximately $3 \mathrm{~km}$ high and $30 \mathrm{~km}$ horizontally, perpendicular to the instrument line-of-sight. The FOV is modelled by a trapezoidal response function $\phi(z)$, having a $4 \mathrm{~km}$-high base and a $2.8 \mathrm{~km}$-high top.

\section{Current detection methods for MIPAS}

The presence of cloud particles in the FOV of infrared remote sounding instruments influences observations registered, due to extraneous absorption, emission and scattering features in a large range of wavelengths. Clouds in the line-of-sight can act as grey-bodies with significant opacity which alter the measured radiation, and introduce serious problems in sensing atmospheric temperatures and gas profiles below the cloud level. All clouds cause a broadband increase in the radiance emitted and measured in the FOV - however thin clouds also introduce a multiple-scattering effect which implies that the instrument measures radiance from below the tangent height. The presence of clouds introduces problems with regular constituent retrievals by introducing a sharp transition from optically thin to optically thick limb transmittance at the cloud top. In order to avoid this, and to maintain retrieval quality/reliability, routine processing of MIPAS spectra includes the detection and rejection of all spectra with significant cloud contamination. However, studying these cloud-contaminated spectra can reveal information about the cloud itself.

The following sections outline the detection methods which have been either proposed or operationally used to detect cloud in MIPAS measurements.

\subsection{Mean Radiance Thresholding and Colour Indices}

A very basic method is the Mean Radiance Threshold test which simply uses a statistically gathered radiance threshold to detect cloud by assuming that clouds have a warmer brightness temperature than a clear limb view. For MIPAS, considering the region around $960.7 \mathrm{~cm}^{-1}$ a radiative transfer model, the Reference Forward Model (the RFM, a GENLN2based line-by-line radiative transfer code originally developed to provide reference spectral calculations for MIPAS by Dudhia, 2005), is used to simulate the transmittance spectrum - and the $960.7 \mathrm{~cm}^{-1}$ region is used as it has high trans-

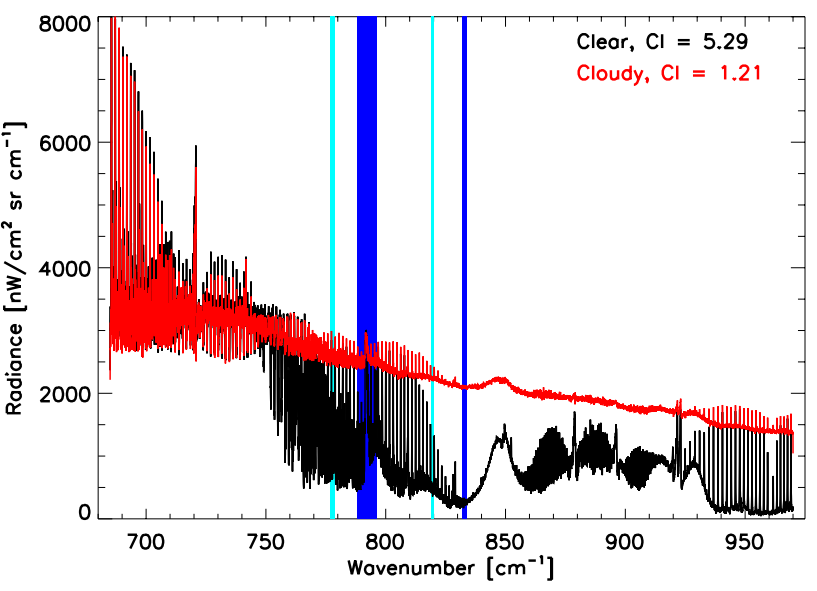

Fig. 1. Samples of clear (black) and cloudy (red) MIPAS spectra with the locations of the current CI MWs (blue) and optimised CI MWs (aqua) overplotted.

mission and low gaseous emission. Thus, in order to detect a cloud having an extinction coefficient of $10^{-4} \mathrm{~km}^{-1}$, a threshold of $100 \mathrm{nW}\left(\mathrm{cm}^{2} \mathrm{srcm}^{-1}\right)^{-1}$ must be chosen at a tangent height of $9 \mathrm{~km}$ (higher threshold for lower tangent heights and for higher extintion values).

A second generation detection method is Colour Index (CI) Thresholding (Spang et al., 2004). CIs work on the principle of radiance ratios between two different regions (called microwindows MWs, and denoted MW1 and MW2) of the spectrum which respond differently to cloud. The MWs are chosen such that the first microwindow MW1 responds very little to the presence of clouds whereas the second microwindow MW2 shows a large reaction, as shown in Fig. 1. Use of a ratio of radiances from each measurement spectrum implies that the variability in radiance resulting from temperature and pressure fluctuations is effectively cancelled out, since both sections of the spectrum will scale consistently to such changes - and hence thresholds can be more reliably picked.

The $\mathrm{CI}$ is defined to be the ratio of the mean radiances of the two MWs:

$\mathrm{CI}=\frac{\bar{L}_{\mathrm{MW} 1}}{\bar{L}_{\mathrm{MW} 2}}$.

When $\mathrm{CI}$ is large $(\mathrm{CI}>4$, for conventionally chosen MWs, $\mathrm{MW} 1=792-796 \mathrm{~cm}^{-1}$ and MW2 $=832-834 \mathrm{~cm}^{-1}$ ), cloudfree conditions exist and when CI is approximately unity optically thick clouds are present. The range of CIs represents the range of optical thickness of clouds present, with thicker clouds appearing blackbody-like with $\mathrm{CI} \approx 1$ and thinner, tenuous clouds registering increasingly larger CIs.

The presence of cloud is then determined by setting a threshold for the CI below which it is said that cloud occurs and above which, cloud is said to not occur. In the interest of 
only conservatively discarding data which are truly contaminated by thick cloud, a low threshold is frequently chosen, below which it is certain that cloud occurs and above which cloud is said to not occur, even though it is well known that above this threshold cloud can indeed occur, either as an optically thin cloud or by only partially filling the instrument FOV. In operational processing for MIPAS, this threshold is set at $\mathrm{CI}=1.8$ (Spang et al., 2004; Ewen, 2005).

It should be noted that the definition of CI breaks down above about $30 \mathrm{~km}$ due to decreased signal-to-noise-ratio, particularly in the more transparent and intrinsically noisier (due to smaller signal) second MW. Cloud detection itself gives a measure of the cloud top height, but this is limited to the height resolution of the measurement scan pattern.

\subsubsection{Analysis of current operational CI detection method}

A useful quantity to measure the amount of cloud present in a measurement FOV is the cloud effective fraction (EF), as defined by

$\mathrm{EF}=\frac{\int_{-d}^{z_{c t}}\left(1-e^{-k_{\mathrm{ext} x}}\right) \phi(z) d z}{\int_{-d}^{d} \phi(z) d z}$

for a FOV of width $2 d$ characterised by the FOV function $\phi(z)$ corresponding to integrated pencil beam radiances each penetrating a pathlength $x$ through an atmosphere of extinction coefficient $k_{\text {ext }}$ and cloud top height $z_{c t}$ relative to the tangent height. It is essentially the effective blocking power of the cloud within the FOV - the proportion of the FOV filled by cloud modified by the extinction of the cloud. Therefore, an $\mathrm{EF}=0$ indicates that a measurement is cloudfree or "clear", an $E F=1$ represents a FOV that is completely filled with thick cloud and $0<\mathrm{EF}<1$ represents the spread of varying cloud-filled states of a FOV.

It can be asserted that the CI Method used operationally fails to detect many cloudy FOVs - as well as incorrectly diagnosing clear spectra as cloudy. Considering the average radiance measured in the $960.0-961.0 \mathrm{~cm}^{-1} \mathrm{MW}$ (a region of the A band spectrum having comparatively high transmissivity - which implies that most variations in radiance come from continuum features, such as induced by clouds) as shown in Fig. 2 (with different values of CI assigned different colours), there exist two distinct regions, one corresponding to cloudy measurements and the other to clear measurements. The leftmost region is a thick band extending through all altitudes at relatively low radiances, which represents the clear measurements. To the right of this thick band is a scattering of radiance points, starting at an altitude that could be taken as the maximum average tropopause height, at higher radiances - these points represent the cloudy measurements. The spread in these cloudy radiances is a result of many possible fractions of cloud experienced by the measurement FOV. The present MWs and threshold used for cloud detection do

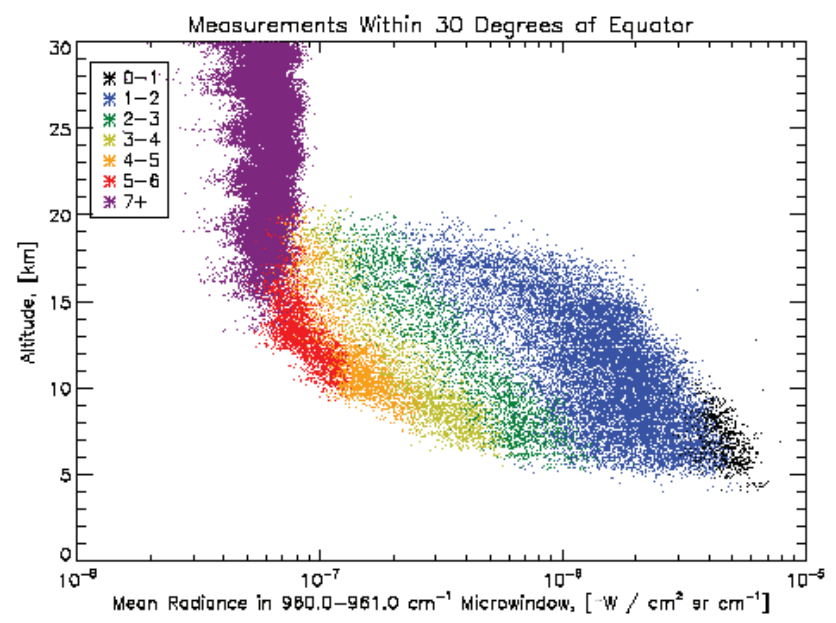

Fig. 2. Average radiance profiles measured in the 960.0$961.0 \mathrm{~cm}^{-1}$ MW by MIPAS, whereby the measurements have been assigned colours to indicate their CI value.

not detect the measurements which through this analysis are obviously cloud-contaminated (i.e. spread to the right of the thick band of clear measurements), although with a small EF. It is interesting to note that increasing the value of the threshold to a higher value of CI (than the currently used 1.8) does have the effect of picking up this scattering of cloudy cases, but that it also results in the clear cases (those measurements occurring in the thick leftmost band) being flagged as cloud as well. Fig. 3 shows that for EF less than about 0.3, the CI Method with the current threshold of 1.8 is not able to detect cloud at all. Altitude-dependent thresholds would partially solve the problem of misdetection, however given the inclusion of clear spectra as cloudy as a consequence of emission by water vapour, the CI method has key caveats which cannot be rectified by simply setting different thresholds.

Furthermore, there is a known problem with the CI method whereby clear spectra are misdiagnosed as cloudy, deriving from the fact that water vapour emissions in the lower atmosphere can create broadband continua features, much like those exploited in the CI method itself (Spang et al., 2004).

Given the relative insensitivity of the current operational cloud detection method to optically thin cloud or of FOVs only partially covered in cloud, as well as its sensitivity to water vapour emissions in the lower atmosphere, there appears to be room for development of a cloud detection method which is capable of reliably resolving and identifying even these small amounts of cloud in measurements.

\subsection{Singular Vector Decomposition}

Singular Vector Decomposition (SVD) is a standard statistical technique (Press et al., 2007) used for finding patterns in high dimensional data and for summarising these data. To this end, SVD transforms a number of potentially correlated 


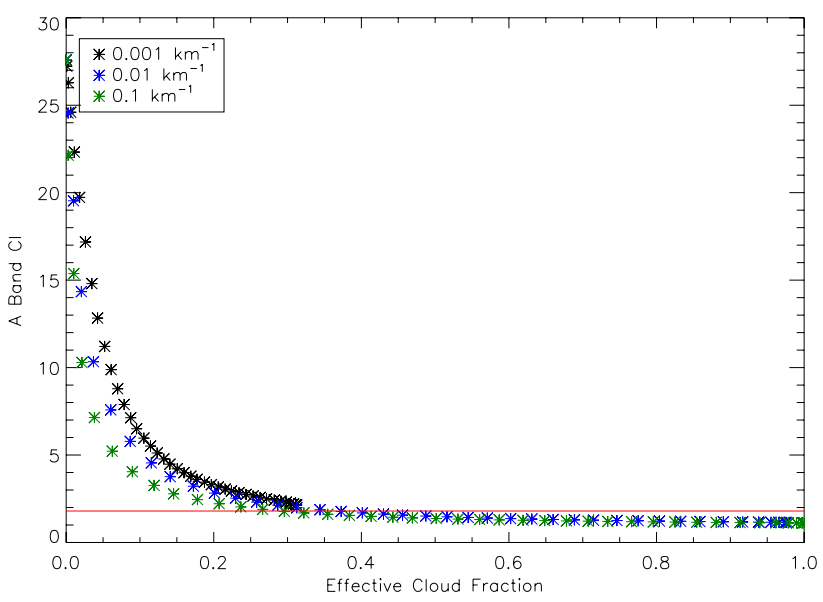

Fig. 3. Relation between cloud effective fraction and CI for RFMsimulated clouds with extinction coefficients of $0.001-0.1 \mathrm{~km}^{-1}$ in the MIPAS A band. The red line shows the CI threshold (1.8) below which cloud is detected.

variables into a smaller number of uncorrelated variables called singular vectors $\boldsymbol{v}$. The first singular vector $\boldsymbol{v}_{0}$ accounts for as much of the variability in the data as possible, and then each successive $\boldsymbol{v}_{i}$ accounts for as much of the remaining variability as possible.

Consider an $m \times n$ matrix $\mathbf{L}$ (Press et al., 2007). In this application, $\mathbf{L}$ is a set of $m$ spectra each of length $n-$ and each spectrum of length $n$ is denoted $\mathbf{I})$. Then, $\mathbf{L}$ can be expressed as

$\mathbf{L}=\mathbf{W}^{T} \mathbf{S V}$

whereby $\mathbf{V}$ and $\mathbf{W}$ are the $m \times n$ and $m \times m$ orthogonal matrices containing left- and right-singular vectors, respectively, and $\mathbf{S}$ is a $m \times m$ diagonal matrix whose diagonal elements contain the $m$ singular values $S_{i}$. The singular values (S) are essentially eigenvalues corresponding to the singular vectors $(v s)$, which are analogous to eigenvectors. Hence, the original matrix $\mathbf{L}$ is merely a linear combination of the singular vectors as scaled by the singular values (Murtagh and Heck, 1987).

Application of the decomposition yields a set $(\mathbf{V})$ of a maximum of $m$ singular vectors ( $\boldsymbol{v} s$ ) each of length $n$ which best orthogonally span the variance of the initial ensemble of measurements (in the sense that the $\boldsymbol{v} s$ can then be thought of as a set of basis vectors in $\mathbb{R}^{n}$ chosen so that the maximum object-to-object variation in the data belongs to a subspace formed by the least number of basis vectors). The vs are usually ordered (by choice) by decreasing magnitude of their eigenvalue. Thus, each successive $v$ captures increasingly less and less information, such that the percentage of the total variance $P_{i}$ captured by the $i$ th $v$ is

$P_{i}=\frac{d_{i}}{\sum_{i=1}^{m} d_{i}} \times 100 \%$.
The original input measurements $\mathbf{L}_{i j}$ can be reconstructed simply by calculating the appropriate linear combination of the $v s$ and their corresponding singulars values, as described by

$\mathbf{L}_{i j}=\mathbf{W}_{i k}^{T} \mathbf{S}_{k l} \mathbf{V}_{l j}$

where $\mathbf{L}_{i j}$ is the $j$ th spectral measurement of the $i$ th spectrum for $i \in[1, m]$ and $j \in[1, n]$. In this notation, summation occurs over the indices $k$ and $l$, where $k \in[1, m]$ and $l$ $\epsilon[1, m]$. Since the first few $\boldsymbol{v} s$ capture so much of the total variance of the dataset, it is often sufficient to only sum over the first few singular vectors (for example, not from 1 to $m$, but rather from 1 to 2 or 3 ) in order to obtain a reconstruction which is good to within a few percent of the full reconstruction.

The objective of this work is to use SVD techniques to create, implement and validate a reliable cloud detection method. The idea is to create an ensemble of simulated MIPAS spectra (Sect. 4) which contain varying amounts of cloud (because the EF characterising each spectrum will be known for simulated spectra) and then to use this ensemble to obtain singular vectors which correspond to the clear and cloudy atmospheric states (Sects. 5.1-5.2). Once the two orthogonal sets of basis vectors (clear and cloudy) are known, any atmospheric signal should be able to be fit using both sets of vectors, regardless of whether the atmosphere is clear or cloudy (Sect. 6). By using some appropriate parameter related to the fitting process, it should be possible to create a cloud detection method (Sect. 8). Finally, this SVD-based cloud detection method will be compared with the current cloud detection method on a year's worth of MIPAS data (Sect. 8) as well as using a set of simulated data for which the clear/cloudy state is known (as introduced in Sect. 4). It is hypothesised that the increased information gained by using large regions of spectra (such as would be done for SVDbased methods) should lead to more reliable cloud detection than those based upon mean-continuum recognition (such as the CI method). Theoretically, it should avoid the misdetection of regions of high water vapour concentration as cloud, a caveat of the CI method, as the water-vapour continuum features should be well represented in clear atmospheric spectra used in the development of clear basis vectors - however this hypothesis has not been extensively tested in this work.

\section{Ensemble of simulated clear and cloudy MIPAS spectra}

The RFM was used to simulate an ensemble of spectra with varying amounts of cloud (as defined by their extinction coefficients $\left(k_{\text {ext }}\right)$ and cloud top heights $(\mathrm{CTH})$ ) occurring in the MIPAS FOV for all the tangent heights at which cloud is normally expected, for many different atmospheric conditions, assuming horizontal homogeneity and aerosol continua 
Table 1. Parameters used to create ensemble of cloudy atmospheres. Reference atmospheres compiled by Remedios (2001).

\begin{tabular}{llll}
\hline $\begin{array}{l}\text { Tangent Height } \\
{[\mathrm{km}]}\end{array}$ & $\begin{array}{l}k_{\mathrm{ext}} \\
{\left[\mathrm{km}^{-1}\right]}\end{array}$ & Reference Atmosphere & $\begin{array}{l}\text { CTH relative to } \\
\text { Tangent Height }[\mathrm{km}]\end{array}$ \\
\hline $6,9,12,15,18,21$ & $0.001,0.01,0.1$ & $\begin{array}{l}\text { standard mid-latitudinal, tropical, polar summer and polar win- } \\
\text { ter reference atmospheres, their one standard-deviational vari- }\end{array}$ & $\begin{array}{l}-2.0,-1.5,-1.0, \\
-0.5,0.0,0.5,1.0,\end{array}$ \\
& & $\begin{array}{l}\text { ants, and separate perturbations in temperature, pressure, water } \\
\text { vapour and ozone of each }\end{array}$ & $1.5,2.0$ \\
& &
\end{tabular}

spectral features. The advantage of having an artificially created ensemble of spectra to examine as opposed to real data is that all of the cloud parameters are known in advance and one can without question identify with confidence different cases and regimes. The parameters used to build this ensemble of spectra are given in Table 1. In total, the ensemble has 5184 different atmospheric conditions: 576 of which are totally clear (i.e. cloud top height $=-2.0 \mathrm{~km}$ ) and 4608 of which which contain some finite amount of cloud in the corresponding MIPAS FOV (here, when cloud top height $>-2.0 \mathrm{~km}$ ). These simulations have been carried out at the MIPAS fullresolution of $0.025 \mathrm{~cm}^{-1}$ in the second half of the MIPAS A band (827.5-970.0 $\mathrm{cm}^{-1}, 5701$ spectral points).

It should be noted that the RFM is a non-scattering model - and hence produces simplified spectra, as real clouds will have both single and multiple scattering features, as well as the broader features reproduced by the RFM. Hurley (2008) used single-scattering simulations to quantify the discrepancy between non-scattering and more-realistic scattering simulations - and found that for clouds having extinction coefficients larger than $10^{-4} \mathrm{~km}^{-1}$, the difference was negligible.

\section{Calculation of singular vectors}

In the following SVD studies, the ensemble discussed in Sect. 4 is separated by tangent height, and data from each tangent height are treated independently. Since, in practise, the nominal tangent height is a well-known discrete parameter of MIPAS data, this segregation has been carried out in order to preserve vertical atmospheric variations which consistently occur.

SVD has been carried out by first dividing the ensemble of spectra into two regimes: clear $(\mathrm{EF}=0)$ and cloudy $(\mathrm{EF} \neq 0)$. Then each of these two atmospheric regimes is sub-divided into smaller ensembles grouped by tangent height. To normalise the data, each spectrum in the ensemble has had its average radiance subtracted (which effectively allows clear and cloudy singular vectors to share reconstructive responsibility of the raised cloud radiance baseline - otherwise the clear singular vectors are simply forced to accommodate more of the radiance coming as a result of the cloud presence, see
Hurley (2008) for details), and then SVD is carried out upon each of the normalised tangent height ensembles.

\subsection{Clear singular vectors}

Using the clear ensemble of spectra, divided by tangent height and normalised, SVD is carried out to calculate the clear singular vectors $\boldsymbol{v}_{\text {clear }_{i}}$. For a tangent height of $9.0 \mathrm{~km}$, Fig. 4 shows the average clear spectrum for the $9.0 \mathrm{~km}$ clear ensemble along with the first eight singular vectors. It should be noted that the zeroth order $\boldsymbol{v}_{\text {clear }}$ carries so much of the variance associated with the ensemble that it visually resembles the average spectrum, while the higher order $\boldsymbol{v}_{\text {clear }} s$ pick up more non-trivial variances, which is expected due to the large range of variations in clear atmospheric spectra due to local changes in pressure and temperature. If the total variance captured by the addition of each successive $\boldsymbol{v}_{\text {clear }}$ in the decomposition is considered, the first three $\boldsymbol{v}_{\text {clear }} s$ contain over $80 \%$ of the total variance. Thus, the SVD method effectively minimises the number of pieces of information needed to represent a set of data, since any of the initial pieces of information (here, the spectra) can be reconstructed by using as few as three singular vectors.

\subsection{Cloudy singular vectors}

Considering now the second ensemble of spectra which consist of simulations of infrared measurements containing some finite amount of cloud, the component of the signal which is due to the cloud alone is sought. The measurement registered by the instrument FOV will be some combination of emission and absorption from the clear atmospheric components (ie. the gases) and those resulting from the cloud presence. The singular vectors obtained for the clear ensemble of spectra should represent the clear component in these mixed clear/cloud measurements and by using these already obtained $\boldsymbol{v}_{\text {clear }} s$, the component due to the cloud alone can be retrieved. The basis of this work is the hypothesis that a cloud-contaminated spectrum can be decomposed into components coming from the clear atmosphere and those due to the cloud itself.

For each tangent height, $m$ clear singular vectors of length $n$ ( $m=96$ and $n=5701$, as before) have been calculated, as described in Sect. 5. If then each cloudy spectrum $\boldsymbol{l}_{\text {cloudy+clear }}$ (which includes, at this point, a clear 

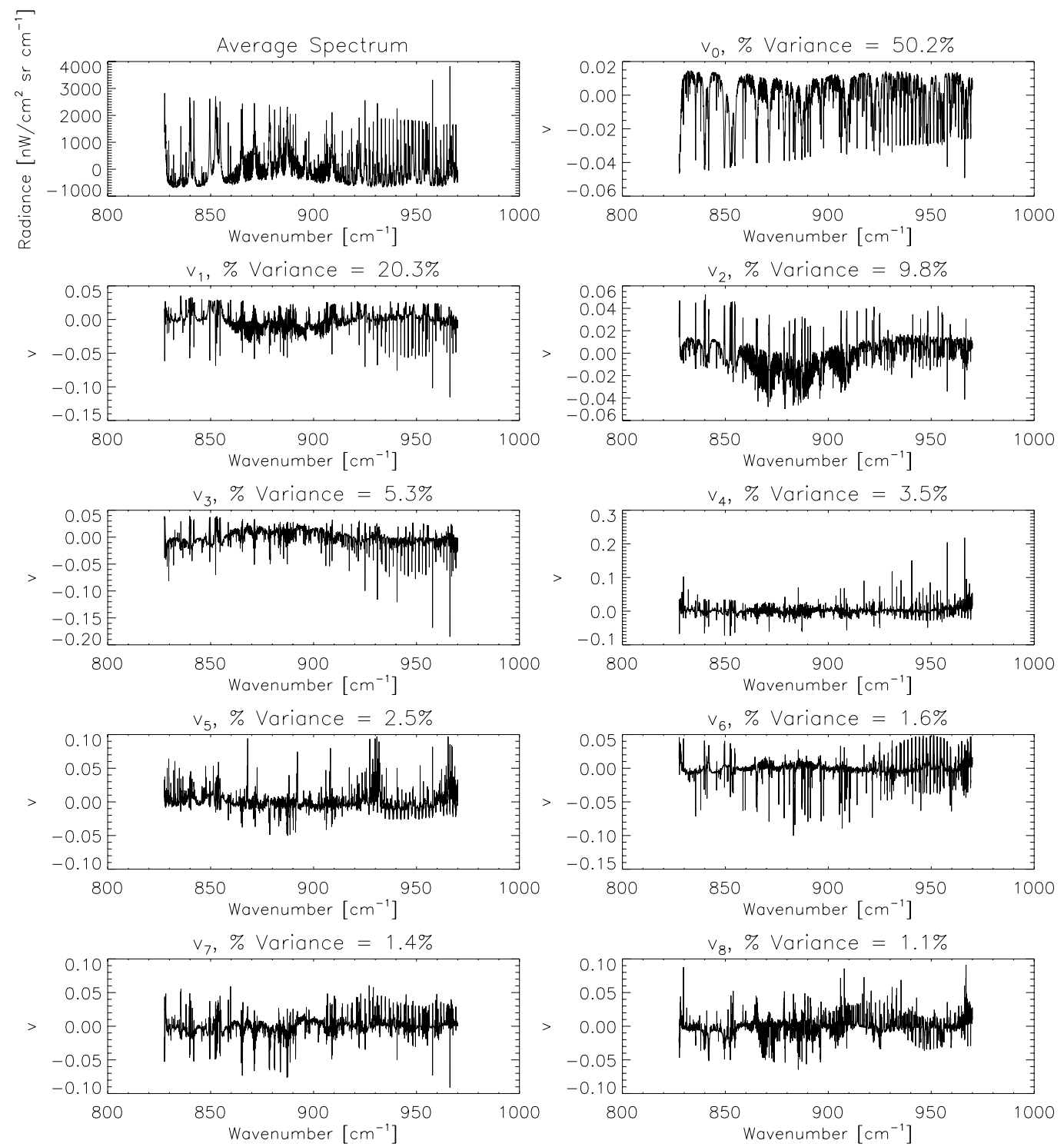

Fig. 4. Average clear spectrum for the $9.0 \mathrm{~km}$ clear ensemble along with the first eight singular vectors and the percentage variance captured by each.

atmospheric contribution along with that coming from the cloud itself) in the cloudy tangent height ensemble is first normalised by subtracting off its average radiance to give

$\boldsymbol{l}_{\text {cloudy }+ \text { clear }_{\text {norm }}}=\boldsymbol{l}_{\text {cloudy }+ \text { clear }}-\overline{\boldsymbol{l}}_{\text {cloudy }+ \text { clear }}$

the component due to the clear background atmosphere can be obtained by carrying out a linear least squares fit using the clear singular vectors $\boldsymbol{v}_{\text {clear }}$ such that the clear radiance component $\boldsymbol{l}_{\text {clear }}$ of the measurement is

$\boldsymbol{l}_{\text {clear }}=\sum_{i=1}^{m} \lambda_{i} \boldsymbol{v}_{\text {clear }_{i}}$ where $\lambda_{i}$ are fit coefficients. Since the fit of the normalised signal by the clear singular vectors will have captured any of the variance due to the clear sky, it is necessary merely to subtract to obtain the cloudy component of the signal $\left(\boldsymbol{l}_{\text {cloudy }}\right)$ :

$l_{\text {cloudy }}=\boldsymbol{l}_{\text {cloudy }+ \text { clear }_{\text {norm }}}-\boldsymbol{l}_{\text {clear }}$.

Carrying out this procedure for each cloudy spectrum in each tangent height ensemble yields an ensemble of spectra registering only the cloudy component for an abundance of cloudy atmospheric conditions. SVD can then be performed on this cloud-signal-only ensemble to yield a set of cloud singular vectors $\boldsymbol{v}_{\text {cloudy }_{i}}$ which are orthogonal to the clear singular 

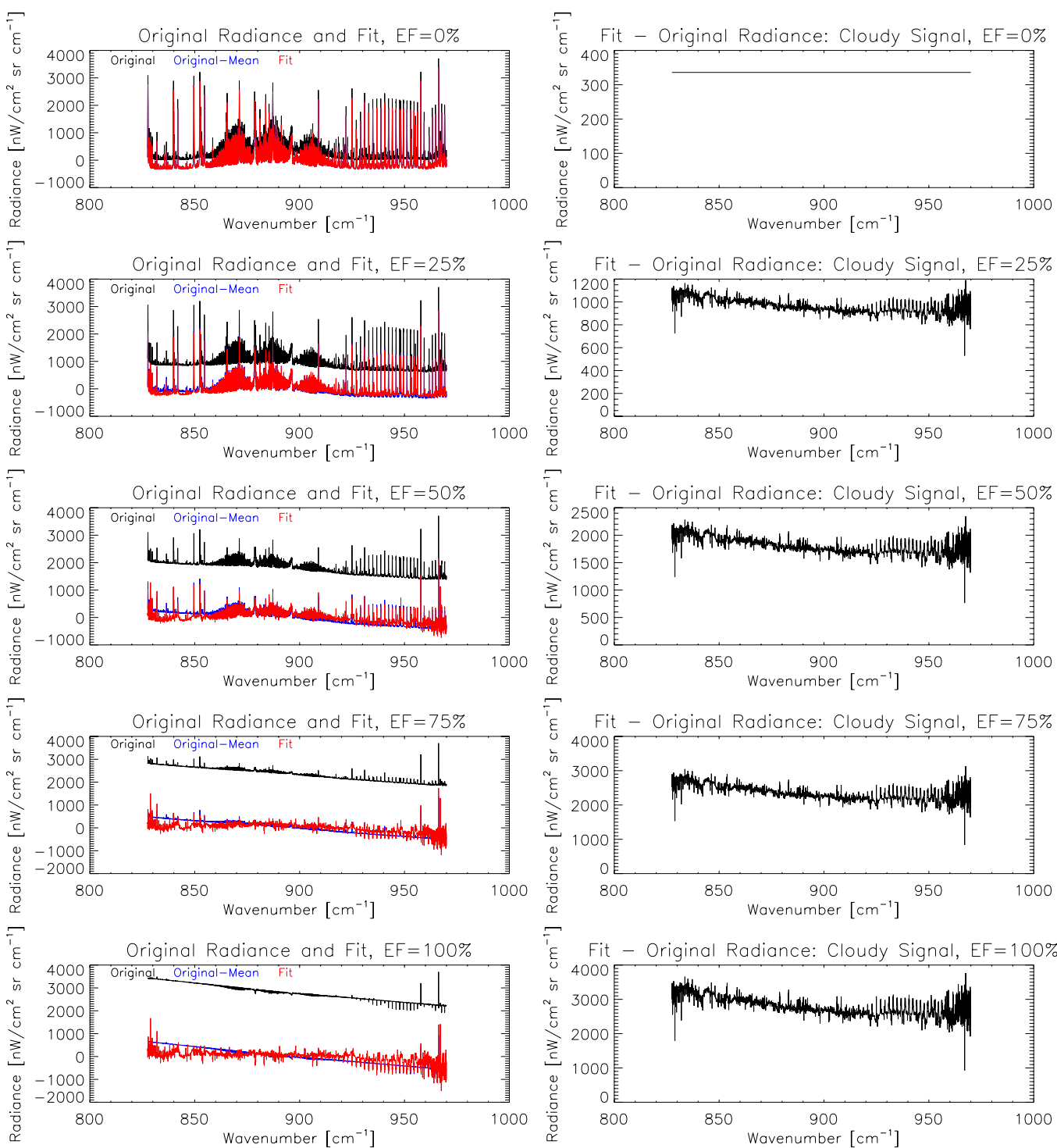

Fig. 5. Fitting of cloudy signal by clear singular vectors to obtain cloud-only signal component for cloud in a $9.0 \mathrm{~km}$ TH FOV. From top to bottom of the plot, EF increases in equal increments of $25 \%$ from $0 \%$ to $100 \%$. Left panels: the original signal containing varying amounts of cloud is shown in black, the normalised original signal in blue, and the clear singular vector least squares fit in red. Right panels: the component of the original signal caused by the cloud as calculated in Sect. 5.2.

vectors $\boldsymbol{v}_{\text {clear }}$. Figure 5 shows how cloudy measurements of varying EF between 0 and 1 can be individually fitted by first normalising the input radiance and then applying the linear least squares fitting in $\boldsymbol{v}_{\text {clear }_{i}}$. It bears noting that the nonzero difference between the linear least squares fit and the original signal is due to the removal of the mean radiance, as expected, and carries no spectral information.

The residual cloudy signal reported is a complicated spectrum with many emission and absorption features which deviate from that of a blackbody at the appropriate cloud top temperature. However, Fig. 6 shows these cloudy signatures compared with blackbody-only signatures (calculated using the Planck function at various wavenumbers), which for most cases, agree well with the baseline of each cloudy signal, so one can be confident that the signals thus obtained are truly due to the presence of cloud alone. The only case in which the blackbody signature deviates from the residual cloudy signal determined here is for the clear case, where the cloudy signal is a non-zero constant (as opposed to the constant zero radiance expected). This is a result of the normalisation procedure used - but will not affect the fitting of arbitrary spectra as it is a constant offset. 

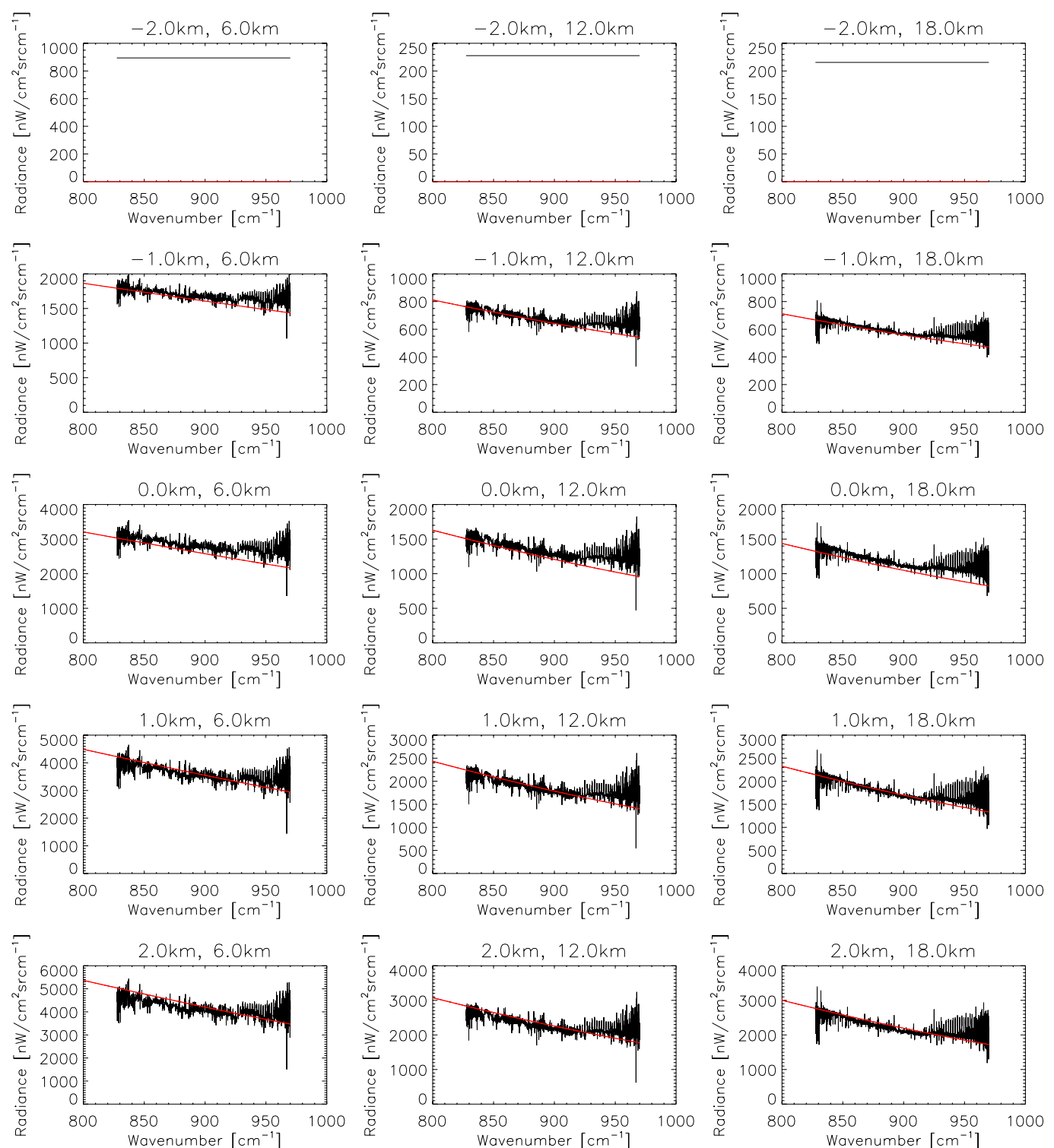

Fig. 6. The cloud-only signal component of original partially cloudy measurement. From top to bottom of the plot, EF increases in equal increments of $25 \%$ from $0 \%$ to $100 \%$. From left to right, the TH of the FOV containing the cloud is increased from $6.0 \mathrm{~km}$ to $12.0 \mathrm{~km}$ to $18.0 \mathrm{~km}$. Blackbody-only signature is overplotted in red and shows good agreement with retrieved cloud-only component of signature given in black.

\section{Fit an arbitrary cloud signal with singular vectors}

Using the previously calculated clear and cloudy singular vectors, $\boldsymbol{v}_{\text {clear }_{i}}$ and $\boldsymbol{v}_{\text {cloudy }_{i}}$ (of which there are $m_{\text {clear }}$ and $m_{\text {cloudy }}$, respectively), for each MIPAS tangent height where cloud is normally expected, any measured MIPAS spectrum in the spectral range of $827.5 \mathrm{~cm}^{-1}$ to $970.0 \mathrm{~cm}^{-1}$ can be accurately fitted by a linear least squares fit in the singular vectors. Taking an arbitrary MIPAS spectrum $\boldsymbol{l}_{\text {orig }}$, the first step is to normalise the spectra by subtracting its average radiance (as explained previously) such that

$$
l_{\text {norm }}=l_{\text {orig }}-\bar{l}_{\text {orig }} \text {. }
$$

The linear least squares fit $\boldsymbol{l}_{\text {fit }}$ of $\boldsymbol{l}_{\text {norm }}$ is then trivially found, such that

$\boldsymbol{l}_{\text {fit }}=\sum_{i=1}^{m_{\text {clear }}} \lambda_{\text {clear }_{i}} \boldsymbol{v}_{\text {clear }}+\sum_{i=1}^{m_{\text {cloudy }}} \lambda_{\text {cloudy }_{i}} \boldsymbol{v}_{\text {cloudy }_{i}}$,

where $\lambda_{\text {clear }_{i}}$ and $\lambda_{\text {cloudy }_{i}}$ are constant coefficients of the least squares fit. In order to regain a fit which can be immediately 
compared with the original input measurement, it remains simply to add back on the constant average radiance of the original signal to the fitted spectra:

$\boldsymbol{l}_{\text {fit orig }}=\boldsymbol{l}_{\text {fit }}+\overline{\boldsymbol{l}}_{\text {orig }}$.

This method was implemented and tested on RFMsimulated spectra for infrared spectra with a tangent height of $9.0 \mathrm{~m}$ and extinction coefficients between $0.001 \mathrm{~km}^{-1}$ and $0.1 \mathrm{~km}^{-1}$ and cloud top heights located every $0.5 \mathrm{~km}$ in the $4.0 \mathrm{~km}$-wide MIPAS FOV. Figure 7 shows the efficiency and consistency with which the linear least squares fit using both the clear and cloudy singular vectors is able to fit a signal with an arbitrary amount of cloud in it for a tangent height of $9.0 \mathrm{~km}$ and extinction coefficient of $0.01 \mathrm{~km}^{-1}$. It is also interesting to note that as increasing amounts of cloud are added to the measurement, the fit coefficients corresponding to the cloudy singular vectors increase in magnitude (particularly that corresponding to the first cloudy singular vector, those of the next first few singular vectors which are nonnegligble, but an order or magnitude smaller than that of the first), while those corresponding to the clear singular vectors decrease in magnitude. This is an encouraging trend, since it is expected that if there is increased cloud presence in the measurement, the signal should be increasingly well fit by the cloudy singular vectors with a minimised dependence upon the clear singular vectors.

This method was then applied to a scan of apodised MIPAS spectra, which has been flagged as cloudy by the CI Method in the final sweep at $6.0 \mathrm{~km}$ but clear everywhere above. Figure 8 shows the fits of the input raw spectra overlaid with the fit obtained from the clear and cloudy singular vectors, which clearly do a good job of fitting the signal since the root mean square error is less than $1.0 \%$ of the measurement's spectral baseline. As well, it is obviously the clear singular vectors which dominate fit until the final sweep, at which the cloudy singular vectors are fitted with non-zero fit coefficients, corresponding well to the present cloud detection mechanism's judgement of the cloudy state of the atmosphere in that sweep only.

Given the success in reproducing spectral features through fitting with the clear and cloudy singular vectors as well as the fact that the clear and cloudy singular vectors are used in relation to each other in a manner which is expected, for both simulated and real MIPAS data in the spectral region considered, it appears as if this method should be able to be used to detect and quantitatively determine the amount of cloud occurring in the MIPAS FOV.

\section{Effect of noise on singular vector fits}

It is interesting to consider how the SVD fit of a noisy signal (such as obtained from real measurements) will differ from that of a noise-free signal. In other words, the way in which the singular value assigned to each noise-free singular vector in the fitting process is affected by noise on the input spectra is sought.

Consider a noise-free radiance spectrum $l$ of length $n$ such that

$\boldsymbol{l}=\left(l_{1}, l_{2}, \cdots, l_{n}\right)$

which is to be fit by a singular vector $\boldsymbol{v}$ of length $n$ where

$\boldsymbol{v}=\left(v_{1}, v_{2}, \cdots, v_{n}\right)$.

Then the least squares linear fit of the spectrum using the singular vector can be expressed as

$\lambda=\left(\boldsymbol{v}^{T} \boldsymbol{v}\right)^{-1} \boldsymbol{v}^{T} \boldsymbol{l}$

It immediately follows that

$\lambda=(\boldsymbol{v} \cdot \boldsymbol{v})^{-1} \boldsymbol{v}^{T} \boldsymbol{l}=(|\boldsymbol{v}|)^{-1} \boldsymbol{v}^{T} \boldsymbol{l}=\boldsymbol{v}^{T} \boldsymbol{l}$

since $|\boldsymbol{v}|=1$ because $\boldsymbol{v}$ is a unit vector by nature. Discretising this yields

$\lambda=\sum_{i=1}^{n} v_{i} l_{i}$

If random noise of amplitude $\sigma$ is added to each spectral point on this arbitrary spectrum, there will be some change $\sigma_{\lambda}$ in the singular value assigned by the least squares fit. The least squares fit to the noisy spectrum can be expressed as

$\lambda_{\text {noisy }}=\lambda+\sigma_{\lambda}=\sum_{i=1}^{n} v_{i}\left(l_{i}+\sigma_{i}\right)$.

It follows that

$\sigma_{\lambda}^{2}=\sum_{i=1}^{n}\left(v_{i}\right)^{2} \sigma^{2}=\sigma^{2} \sum_{i=1}^{n}\left(v_{i}\right)^{2}=\sigma^{2}$

since $\sum_{i=1}^{n}\left(v_{i}\right)^{2}=1$ as $\boldsymbol{v}$ is a unit vector. Hence the fit coefficient to the noisy spectrum is simply

$\lambda_{\text {noisy }}=\lambda+\sigma$.

For MIPAS, $\sigma=50 \mathrm{nW}\left(\mathrm{cm}^{2} \mathrm{srcm}^{-1}\right)^{-1} 1$. Typical fit coefficients for the first few singular vectors in both the clear and cloudy sets (i.e. those important to the fit, as they represent the largest variances) are of the order of $10000 \mathrm{nW}\left(\mathrm{cm}^{2} \mathrm{sr} \mathrm{cm}^{-1}\right)^{-1}$ - so the change in the fit coefficient $\left(\lambda_{\text {noisy }}-\lambda=\sigma\right)$ is minor for most $\lambda$ since $\lambda \gg \sigma$ for most $\boldsymbol{v} s$. Thus, the difference caused by the presence of this maximum value of noise is negligible.

Therefore, random error on the input measurements should not greatly affect the fitting of the spectra by noisefree singular vectors as the vectors important in the fitting mechanism are negligibly changed by the noise.

\footnotetext{
${ }^{1}$ This is an overestimation of the effect of noise, since Eq. (18) assumes no spectral correlation, which is not the case for apodised spectra, as used here. It does represent nevertheless a good upper limit for the effect that noise will have on the fitting process.
} 

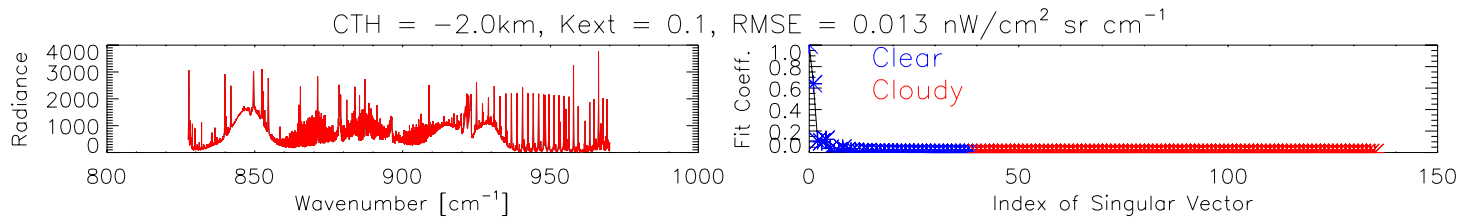

$\mathrm{CTH}=-1.5 \mathrm{~km}$, Kext $=0.1$, RMSE $=0.570 \mathrm{nW} / \mathrm{cm}^{2} \mathrm{sr} \mathrm{cm}^{-1}$
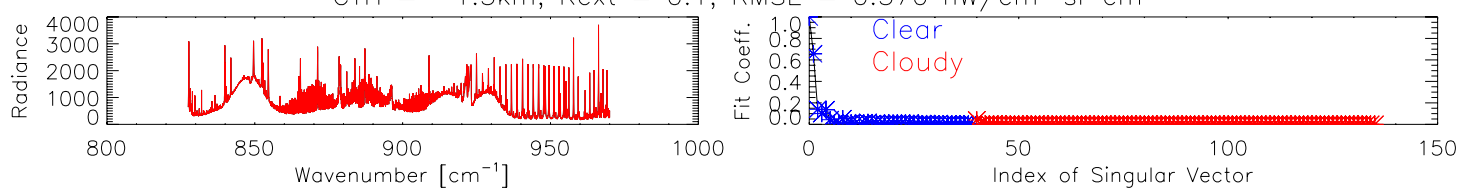

CTH $=-1.0 \mathrm{~km}$, Kext $=0.1$, RMSE $=0.009 \mathrm{nW} / \mathrm{cm}^{2} \mathrm{sr} \mathrm{cm}^{-1}$
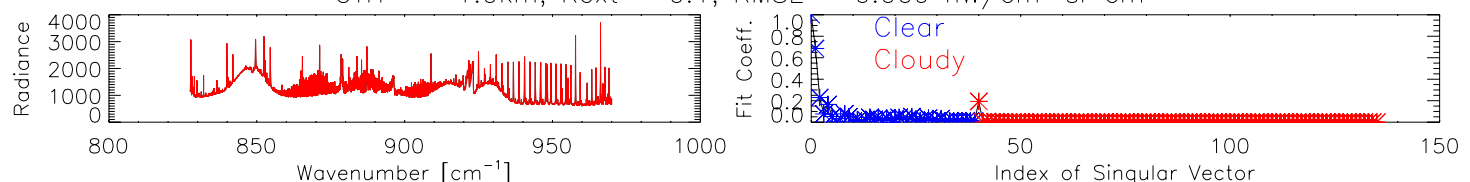

$\mathrm{CTH}=-0.5 \mathrm{~km}$, Kext $=0.1$, RMSE $=0.551 \mathrm{nW} / \mathrm{cm}^{2} \mathrm{sr} \mathrm{cm} \mathrm{cm}^{-1}$
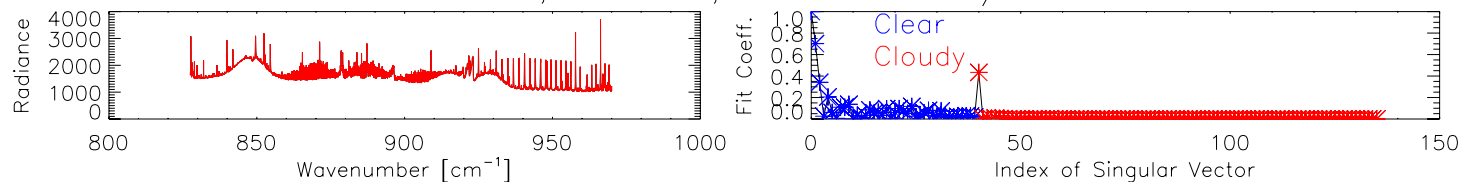

$\mathrm{CTH}=0.0 \mathrm{~km}$, Kext $=0.1$, RMSE $=0.006 \mathrm{nW} / \mathrm{cm}^{2} \mathrm{sr} \mathrm{cm}{ }^{-1}$
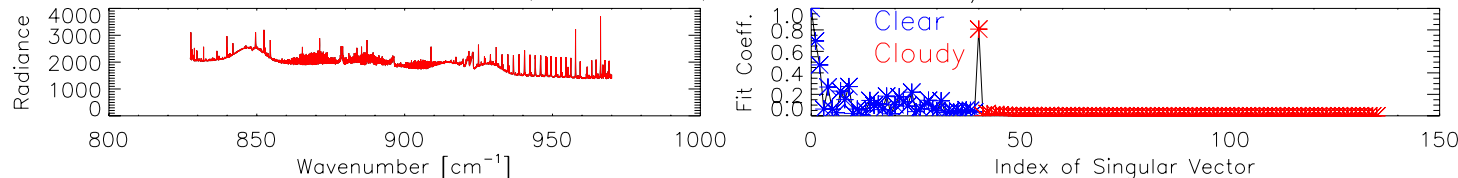

$\mathrm{CTH}=0.5 \mathrm{~km}$, Kext $=0.1$, RMSE $=0.519 \mathrm{nW} / \mathrm{cm}^{2} \mathrm{sr} \mathrm{cm}^{-1}$
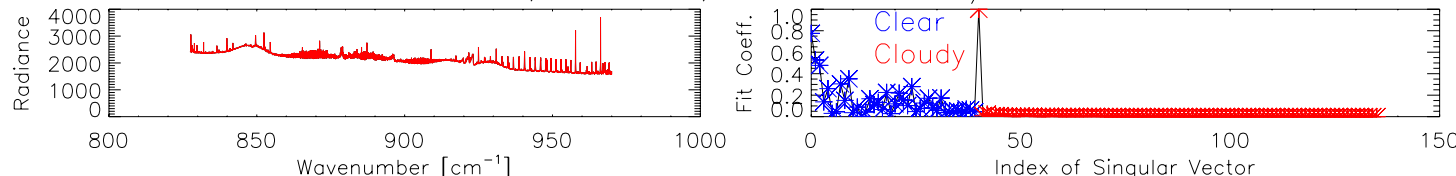

CTH $=1.0 \mathrm{~km}$, Kext $=0.1$, RMSE $=0.006 \mathrm{nW} / \mathrm{cm}^{2} \mathrm{sr} \mathrm{cm}^{-1}$
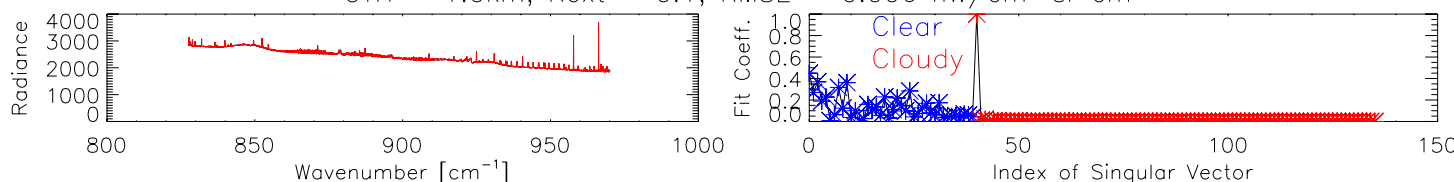

CTH $=1.5 \mathrm{~km}$, Kext $=0.1$, RMSE $=0.528 \mathrm{nW} / \mathrm{cm}^{2} \mathrm{sr} \mathrm{cm}{ }^{-1}$
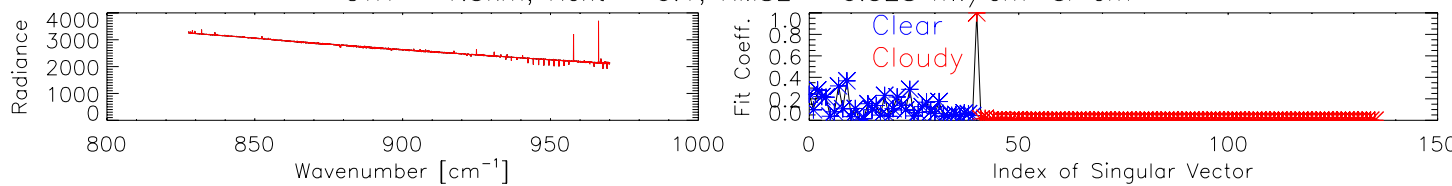

CTH $=2.0 \mathrm{~km}$, Kext $=0.1$, RMSE $=0.008 \mathrm{nW} / \mathrm{cm}^{2} \mathrm{sr} \mathrm{cm}^{-1}$
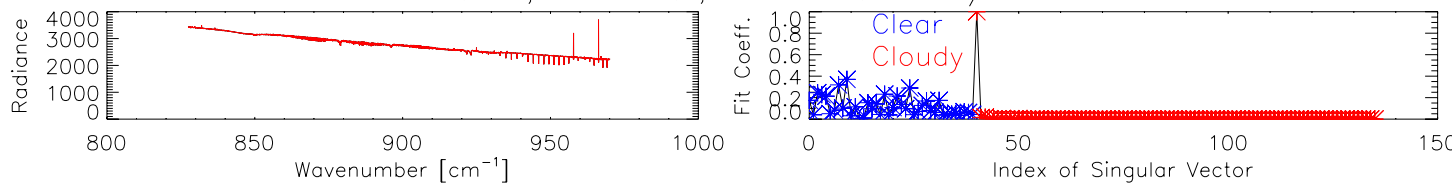

Fig. 7. Left panels: Linear least squares fit using both clear and cloudy singular vectors (red) overplotted on original input signal (black). Right panels: magnitudes of fit coefficients of singular vectors used in the fit (blue=clear, red=cloudy), normalised such that the largest fit coefficient has a magnitude of unity. From top to bottom, the EF increases from $0 \%$ to nearly $100 \%$. 

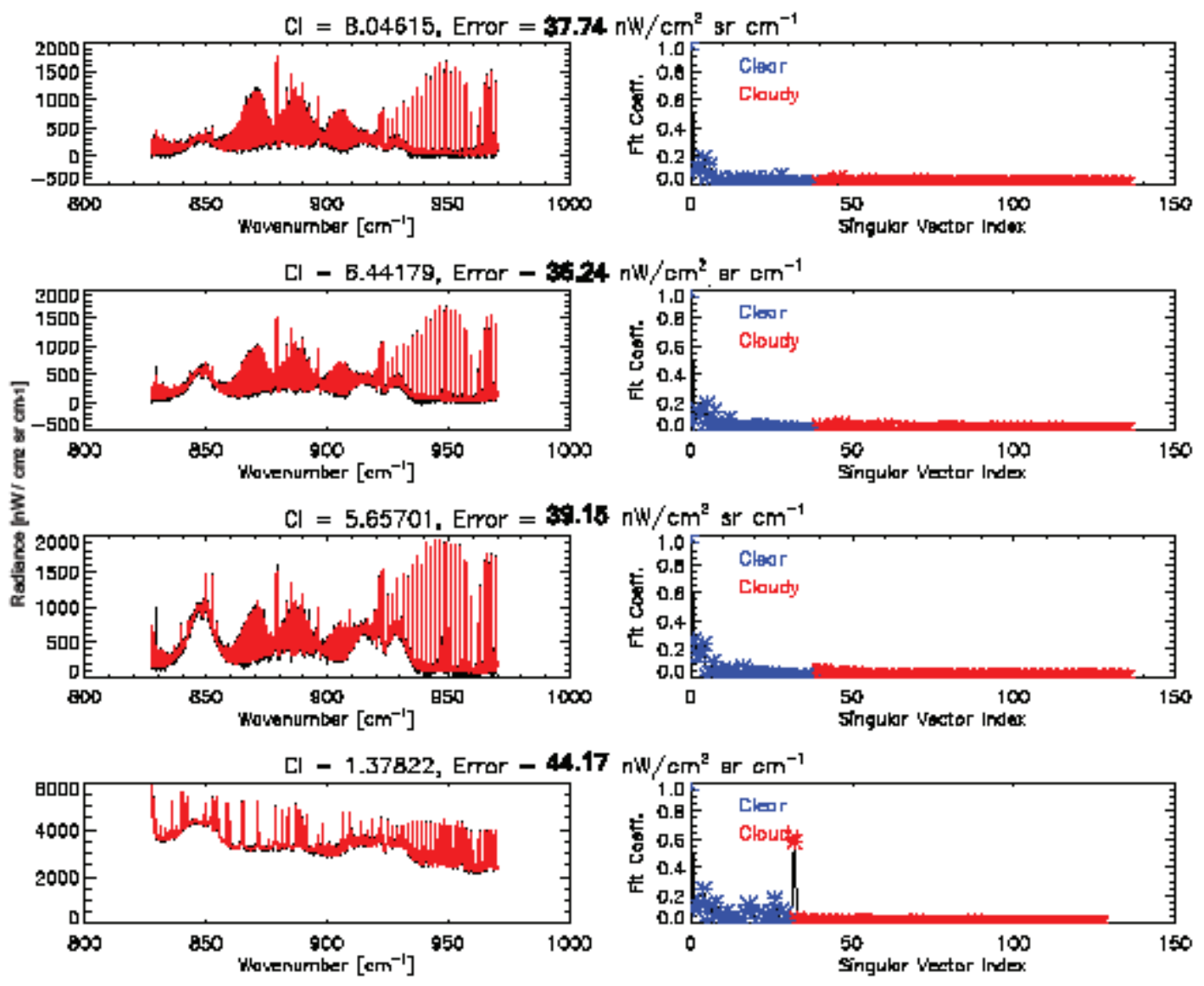

Fig. 8. Fitting of MIPAS spectra by clear and cloudy singular vectors. From top to bottom, downwards through the scan pattern: $15.0 \mathrm{~km}$, $12.0 \mathrm{~km}, 9.0 \mathrm{~km}$ and $6.0 \mathrm{~km}$ tangent heights. Left panels: linear least squares fit using both clear and cloudy singular vectors (red) overplotted on original input signal (black). Right panels: magnitudes of fit coefficients corresponding to the singular vectors used in the fit (clear in blue, cloudy in red), normalised such that the largest fit coefficient has a magnitude of unity.

\section{SVD cloud detection method}

As described in the previous sections, using the set of clear and cloudy singular vectors should yield a cloud detection mechanism. This section will introduce and test a possible candidate for detection mechanism which reconstructs the portion of radiance that the fit attributes to a cloudy presence.

Any arbitrary spectrum can be successfully fit to a high degree by a set of altitude-dependent singular vectors which span the clear and cloudy atmospheric states such that

$\boldsymbol{l}_{\text {total }}=\sum_{i=1}^{m_{\text {clear }}} \lambda_{\text {clear }_{i}} \boldsymbol{v}_{\text {clear }_{i}}+\sum_{i=1}^{m_{\text {cloudy }}} \lambda_{\text {cloudy }_{i}} \boldsymbol{v}_{\text {cloudy }_{i}}$

in keeping with standard reconstruction of SVD, as discussed in Eq. (5), where $\lambda_{\text {clear }_{i}}$ and $\lambda_{\text {cloudy }_{i}}$ are constant coefficients of the least squares fit. Once this linear least squares fit has been obtained, it is trivial to reconstruct the radiance components of the original signal: that due to the clear background state and that due to possible cloud presence. Reconstructing, the clear radiance is

$\boldsymbol{l}_{\text {clear }}=\sum_{i=1}^{m_{\text {clear }}} \lambda_{\text {clear }_{i}} \boldsymbol{v}_{\text {clear }_{i}}$,

and the radiance due to the cloud presence is

$\boldsymbol{l}_{\text {cloudy }}=\sum_{i=1}^{m_{\text {cloudy }}} \lambda_{\text {cloudy }_{i}} \boldsymbol{v}_{\text {cloudy }_{i}}$.

It follows, then, that when the radiance due to cloud presence becomes non-zero, cloud is present. To normalise this quantity, the ratio of the cloudy radiance to the total radiance $L_{\text {total }}$, called the Integrated Radiance Ratio, is considered such that

$\frac{\bar{L}_{\text {cloudy }}}{\bar{L}_{\text {total }}}>0$

for cloudy spectra and

$\frac{\bar{L}_{\text {cloudy }}}{\bar{L}_{\text {total }}} \approx 0$ 

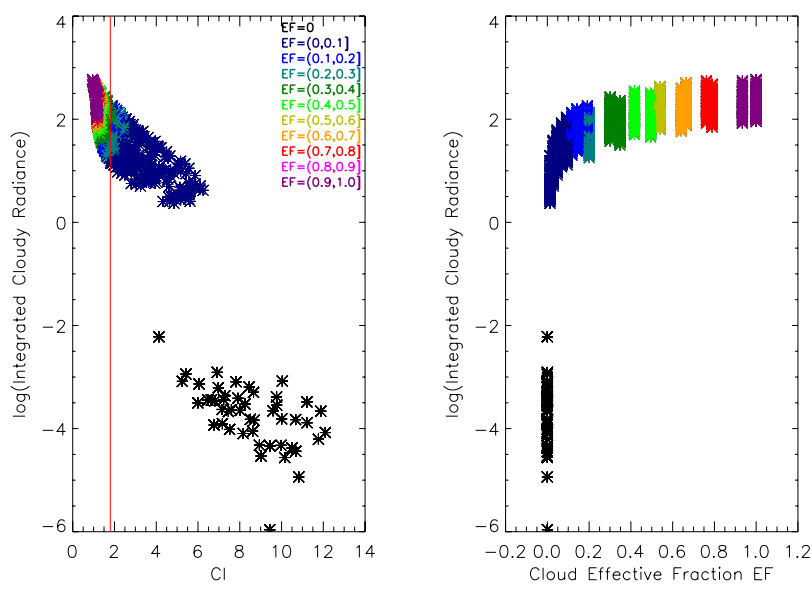

Fig. 9. Integrated Radiance Ratio for all RFM-simulated spectra in ensemble plotted as a function of CI (left panel) and EF. Colourcoded by EF.

for clear spectra, where $\bar{L}$ represents the average of the reconstructed radiance $l$ in the $960-961 \mathrm{~cm}^{-1}$ MW. It is hypothesised that this ratio could be used as a cloud detection method.

The ensemble of RFM-simulated MIPAS spectra has again been used to test this hypothesis. Following the least squares fitting of each spectrum with the altitude-corresponding set of clear and cloudy singular vectors, the cloudy radiance is reconstructed as previously described, the average in the $960-961 \mathrm{~cm}^{-1}$ region calculated and the Integrated Radiance Ratio determined. When the ratio is plotted against CI or EF, as shown in Fig. 9, it becomes obvious that this hypothesis is valid, as the ratios form a bimodal distribution corresponding to clear and cloudy cases.

Thus, the Integrated Radiance Ratio is calculated for all MIPAS spectra measured for the full year of 2003 between $60^{\circ} \mathrm{S}$ and $60^{\circ} \mathrm{N}$. To confidently choose thresholds, it is a matter of fitting the clear peak in the bimodal distribution to a Gaussian distribution - however this is not a trivial procedure since above the clear distribution maximum, there will be non-negligible cloud cases from the tailing edge of the cloudy portion of the overall distribution. Therefore, in fitting the clear distribution, only points in the distribution occurring to the left of the peak are considered. Furthermore, the thresholds are altitude-dependent and will be assigned for each unit altitude between $6.0 \mathrm{~km}$ and $21.0 \mathrm{~km}$. In this manner, probability distributions functions corresponding to each unit altitude between $6.0 \mathrm{~km}$ and $21.0 \mathrm{~km}$ are considered and the "clear" peak (that centred the furthest to the left) fitted by a Gaussian distribution and the altitude-dependent threshold set at

$\operatorname{Thr}(z)=\mu(z)+3 \sigma(z)$, for the peak maximum $\mu$ and standard deviation $\sigma$. Figure 10 shows the PDFs, overplotted with the clear peak fit with $\mu$ and $\sigma$ noted. It is reassuring to note that at the higher considered altitudes, the cloudy peak in the bimodal distribution becomes negligible with infrequent cloud expected. It should be noted that the thresholds thus chosen for the lowest altitudes should be treated with some care, as there is significant overlap between the clear-and-cloudy distributions which may not be fully isolated in the estimation of thresholds.

\section{Application to MIPAS data}

This SVD detection method, with the previously derived thresholds, is applied to all available MIPAS data from 2003, as shown in Fig. 11. It appears that this Integrated Radiance Ratio cloud detection method does a good job in identifying even the thin cloud that the present CI Method appears to miss, choosing all points to the right of the thick clear band in Fig. 11 as cloud - and thus to a first order, it appears to do better than the existing CI Method in terms of identifying cloud. The SVD method suggests that there are $28 \%$ of scans having cloud occurrence somewhere in the altitude range ubiquitous with high cloud (6-24 km), comparing well with Wylie et al.'s (2005) result which records high cloud in $33 \%$ of measurements taken (the CI method sees only $17 \%$ of scans as cloud-filled, with its current thresholds).

It is important to note that detection methods are highly sensitive to choice of threshold, although the choice of threshold is an imminently important component of the detection method itself. It could be argued that the operational CI method has been developped to identify thick cloud in order to avoid spurious trace species retrievals, and hence the operational thresholds are not tailored to isolate thin cloud, whilst the SVD method has been so developed. Extensive comparisons of the two methods are available in Hurley (2008) but have not been presented here for the sake of brevity (employing simulated clear and cloudy MIPAS spectra, as well as real MIPAS spectra, using a wide-range of tests with-and-without the application of thresholds in order to examine the intrinsic skill of detection of the methods).

\section{Application to polar stratospheric clouds}

It should be noted that the SVD detection method has been developed specific to tropospheric clouds only - and that it has not been made to represent (in more than basic widescale continuum cloud features) stratospheric clouds such as polar stratospheric clouds (PSCs), as it was felt that the RFM could not provide spectra representative of PSCs - and at the time that this work was carried out, there existed no database of PSC-specific spectra for MIPAS. However, there now exists such a database, available from the MIPclouds project and detailed in Spang et al. (2008). A natural progression of 

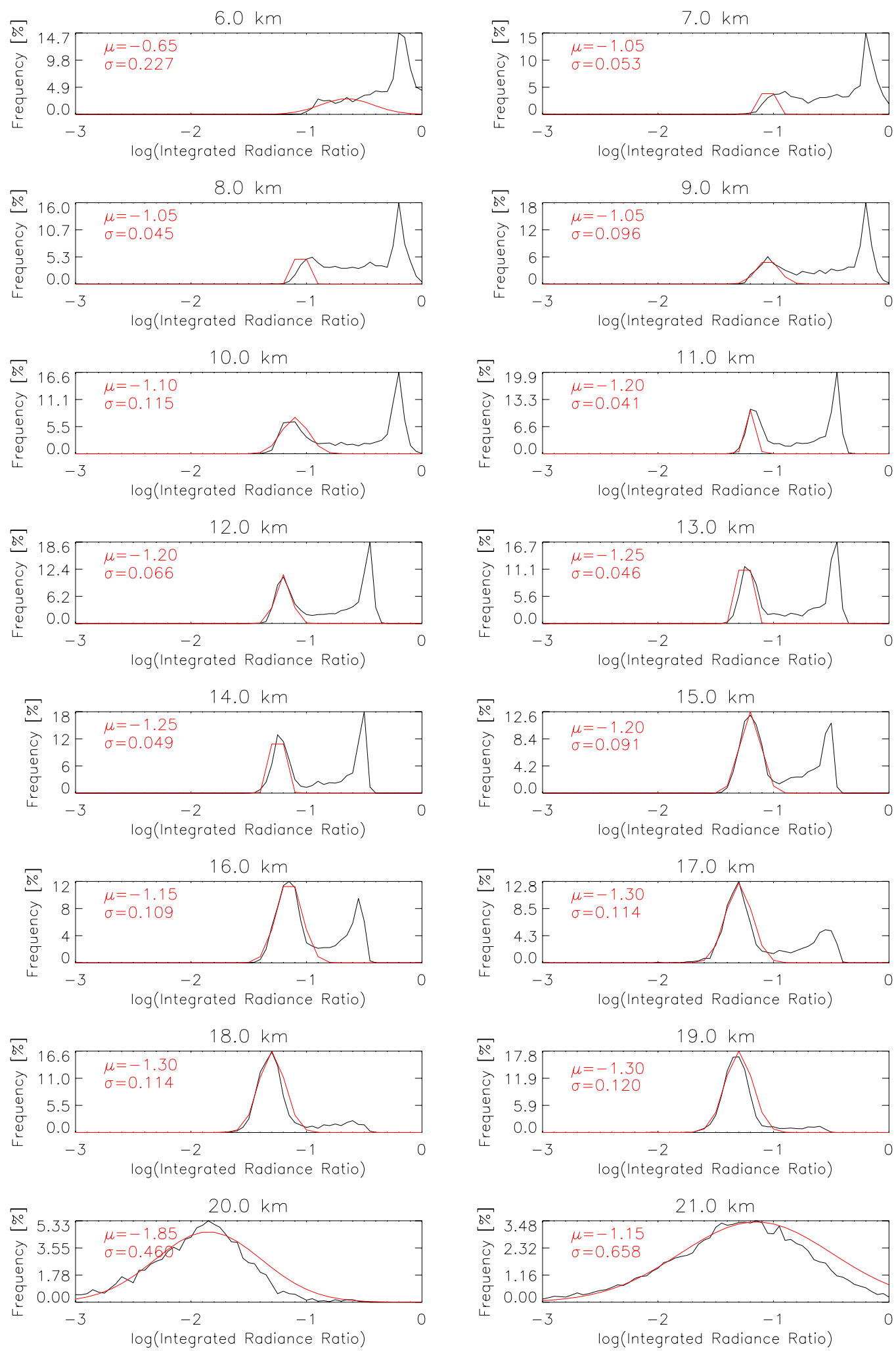

Fig. 10. Altitude-dependent PDFs of $\log \left(\frac{L_{\text {cloudy }}}{L_{\text {total }}}\right)$ for all MIPAS spectra taken in 2003 beween $60^{\circ} \mathrm{S}$ and $60^{\circ} \mathrm{N}$. Each is plotted separately, with the Gaussian fit of the clear distribution overplotted with maxima and standard deviations noted. 

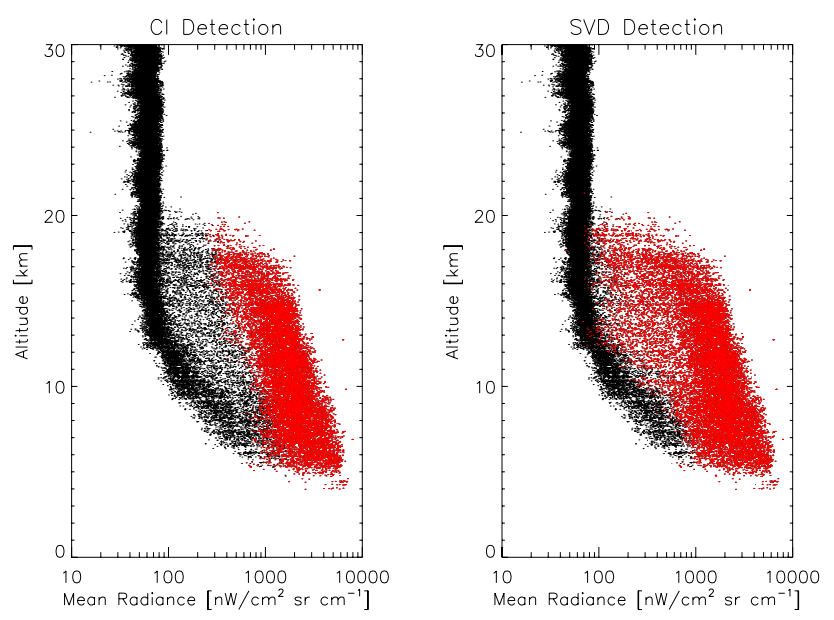

Fig. 11. Profiles of average radiance in $960.0-961.0 \mathrm{~cm}^{-1} \mathrm{MW}$ from all MIPAS spectra taken in 2003, between $60 \mathrm{~S}$ and $60 \mathrm{~N}$. Left panel indicates in red those cases flagged as cloud by the CI Method. Right panel indicates in red those cases flagged as cloud by SVD Integrated Radiance Ratio Method.

this work is indeed to use this database to define basis vectors specific to PSCs, and to perhaps extend this detection method to differentiate between PSC types - a preliminary study of which was carried out in Hurley (2008) using real MIPAS PSC spectra.

\section{Conclusions}

SVD has been applied to an ensemble of simulated spectra which represent a large number of atmospheric states, both clear and cloudy. Singular vectors have been calculated which span both clear and cloudy atmospheres - and a cloud detection method (Integrated Radiance Ratio) has been formulated and tested, exploiting statistics of linear combinations of the two sets of singular vectors to represent any spectra encountered. Appropriate thresholds have been chosen by application to MIPAS data from 2003, and the methods qualitatively tested on MIPAS data from 2003.

It appears that broadband spectral information can be extracted by SVD and used reliably to detect cloud. The true success of this analysis lies in the apparent improvement that the SVD detection method seem to have over the operationally used CI method in the detection of thin cloud.

Simulated spectra have been used in the development of this analysis, which, arguably may not represent all of the possible clear atmospheric states - nor all the cloudy iterations. An interesting exercise would be to form singular vectors from real MIPAS spectra, however this poses the difficulty of not knowing whether or not a singular vector corresponds to the clear atmosphere, or to a cloudy one. Whilst there may be bifurcations in the appropriate distributions of singular values, this is likely not a trivial task. In any case, the simulated singular vectors appear to do a good job at representing the real atmosphere - and the suggested detection methods seem to pick up both simulated cloud and what is hypothesised to be cloud in the real measurements.

Acknowledgements. This work was done as part of a DPhil undertaken at the University of Oxford under the funding of the Commonwealth Scholarship Committee in the UK.

Edited by: B. Mayer

\section{References}

Barton, I.: Upper-level cloud climatology from an orbiting satellite, J. Atmos. Sci., 40, 435-447, 1983.

Dudhia, A.: The Reference Forward Model (RFM), Software User's Manual (SUM), http://www.atm.ox.ac.uk/RFM/sum/ (last access: September 2009), 2005.

ESA ENVISAT website: http://envisat.esa.int/instruments/images/ MIPAS_Interferometer.gif (last access: June 2006), 2005.

Ewen, G.: Infrared Limb Observations of Cloud, DPhil thesis in Atmospheric, Oceanic and Planetary Physics, University of Oxford, Oxford, UK, 2005.

Hurley, J.: Detection and Retrieval of Clouds from MIPAS, thesis in partial fulfillment of DPhil in Atmospheric, Oceanic and Planetary Physics, University of Oxford, Oxford, UK, 2008.

ISCCP website: http://isccp.giss.nasa.gov/index.html (last access: September 2008), 2008.

Mantovani, R.: ENVISAT MIPAS Report: Mar 2004-Feb 2005, ENVI-SPPA-EOPG-TN-05-0006, 2005.

Murtagh, F. and Heck, A.: Multivariate Data Analysis, D. Reidel Publishing Company, Dordrecht, Holland, 1987.

Prabhakara, C., Fraser, R., Dalu, G., Wu, M., Curran, R., and Styles, T.: Thin cirrus clouds: Seasonal distribution over oceans deduced from NIMBUS-4 IRIS, J. Appl. Meteor., 27, 379-399, 1988.

Press, W., Teukolsky, S., Vettering, W., and Flannery, B.: Numerical Recipes: The Art of Scientific Computing, Cambridge University Press, 3, 65-74, 2007.

Remedios, J.: Profiles for MIPAS, EOS, Space Research Centre, Leicester, UK, January 2001.

Sayer, A., Campmany, E., Dean, S., Ewen, G., Poulsen, C. A., Arnold, C., Thomas, G. E., Grainger, R. G., Siddans, R., Lawrence, B., and Watts, P.: Validation of GRAPE ORAC ATSR-2 cloud products, in preparation, 2009.

Spang, R., Remedios, J., and Barkley, M.: Colour Indices for the Detection and Differentiation of Cloud Types in Infra-red Limb Emission Spectra, Adv. Space Res., 33, 1041-1047, 2004.

SAGE III ATBD Team: SAGE III Algorithm Theoretical Basis Document (ATBD) Cloud Data Products, LaRC 475-00-106, 1.2, 2002.

Spang, R., Griessbach, S., Hopfner, M., Dudhia, A., Hurley, J., Siddans, R., Waterfall, A., Remedios, J., and Sembhi, H.: Technical Note: Retrievability of MIPAS cloud parameter, ESA-ITT AO/15255/06/I-OL, 2008.

Warren, S., Hahn, C., and London, J.: Simultaneous occurrence of different cloud types, J. Clim. Appl. Meteot., 24, 658-667, 1985. 
Woodbury, G. and McCormick, M.: Global Distributions of Cirrus Clouds Determined from SAGE Data, Geophys. Res. Lett., 10, 1180-1183, 1983.

Wylie, D. and Menzel, W.: Two years of cloud cover statistics using VAS, J. Clim. Appl. Meteor., 2, 380-392, 1989.
Wylie, D., Menzel, W., Woolf, H., and Strabala, K.: Four Years of Global Cirrus Cloud Statistics Using HIRS, J. Climate, 7, 19721986, 1994.

Wylie, D., Jackson, D., Menzel, W., and Bates, J.: Trends in Global Cloud Cover in Two Decades of HIRS Observations, J. Climate, 130, 18, 3021-3033, 2005. 